\title{
ESTILOS DE ENSEÑANZA Y PROCESOS ATENCIONALES EN NIÑOS EN EDAD ESCOLAR
}

\section{TEACHING STYLES AND CARE PROCESSES IN SCHOOL-AGE CHILDREN}

Gómez-Bohórquez Claudia Pilar , Gutiérrez-Montenegro Yamile, Machado-Osorio Diana Enith, Vicuña-de Rojas Jenny

Volumen 3, 2019.

Enviado: 03/08/2018

Aceptado: 4/2/2019 


\title{
ESTILOS DE ENSEÑANZA Y PROCESOS ATENCIONALES EN NIÑOS EN EDAD ESCOLAR
}

\author{
TEACHING STYLES AND CARE PROCESSES IN SCHOOL-AGE CHILDREN
}

Gómez-Bohórquez Claudia Pilar1, Gutiérrez-Montenegro Yamile2, Machado-Osorio Diana Enith3 , Vicuña-de Rojas Jenny 4

\footnotetext{
1 Licenciada en Educación. Estudiante Especialización Neuropsicopedagogía Infantil. Universidad Católica Luis Amigó, Medellín, Colombia. claudia.gomezbo@amigo.edu.co

2 Licenciada en Educación Preescolar. Estudiante Especialización Neuropsicopedagogía Infantil. Universidad Católica Luis Amigó, Medellín, Colombia. yamile.gutierrez.m@gmail.com

3 Licenciada en Educación Preescolar. Estudiante Especialización Neuropsicopedagogía Infantil. Universidad Católica Luis Amigó. Medellín, Antioquia, Colombia diana.machado1@hotmail.com

4 PhD. en Ciencias de la Educación. Universidad Católica Luis Amigó. Medellín, Colombia. jenny.vicunade@amigo.edu
} 


\section{Resumen}

El propósito de este trabajo consistió en la revisión y análisis de artículos resultados de investigaciones sobre las variables: estilos de enseñanza, estilos de aprendizaje y procesos atencionales. El rastreo se realizó en cuatro bases de datos, con el objetivo de mostrar la posible relación entre las variables y el estado actual de las mismas. Los resultados del análisis dan cuenta de la relación que existe alrededor del rol docente, el cual está atravesado por características personales y comportamentales, y que este incide en la forma en que sus estudiantes procesan,

comprenden, memorizan, integran y aplican conocimientos.

Palabras clave: estilos de enseñanza, estilos de aprendizaje, procesos atencionales, rendimiento académico.
The purpose of this work was to review and analyze the results of research on the variables: teaching styles, learning styles and attentional processes. The tracking was carried out in 4 databases, in order to show the possible relationship between the variables and their current status. The results of the analysis of the sources of documentary review show the relationship that exists around the teaching role that is crossed by the personal and behavioral characteristics, and that this affects the way in which the students process, understand, memorize, integrate and It affects knowledge.

Keywords: teaching styles, learning styles and attentional processes, academic performance. 


\section{INTRODUCCIÓN}

En la literatura científica los conceptos sobre estilos de enseñanza, estilos de aprendizaje y procesos atencionales parecen definirse de acuerdo a la perspectiva personal de cada autor, sin consenso a la hora de abordar y nombrar los términos para referirse a conceptos similares. Algunas definiciones con respecto a cada una de las tres variables a pesar de tener similitud aparecen nombradas de manera diferente aunque se refieren al mismo concepto. Esto por supuesto se convierte en una limitación a la hora de indagar, rastrear y clasificar las investigaciones producidas alrededor de dichos temas.

Hoy en día frente a los avances de la sociedad, donde el conocimiento viene cobrando gran relevancia al punto de convertirse en una "sociedad científica, academizada" (Krüger, 2006) y lo que esta realidad impone al docente, se asume que la mejora de cualquiera de las actuaciones humanas pasa por el conocimiento y el control de las variables que intervienen en ellas. Lo que coloca en evidencia complejos procesos de enseñanza y aprendizaje, y por consecuencia requieren de docentes dispuestos a utilizar medios adecuados para la interpretación y solución de lo que sucede dentro del aula.

Desde la práctica pedagógica actual y las exigencias al ejercicio docente, viene tomando auge la reflexión sobre los estilos de enseñanza y los estilos de aprendizaje de los estudiantes, de cómo influye la práctica pedagógica del docente en los resultados que obtiene y sus implicaciones en el aprendizaje del estudiante y en los procesos que se desarrollan en los salones de clase, esto desde el objetivo de formar docentes y estudiantes para una sociedad del conocimiento.

La revisión documental iniciada buscó mostrar el estado actual de la variable estilos de enseñanza, estilos de aprendizaje y procesos atencionales y la posible relación entre ellas. Para ello se realizó un rastreo inicial de artículos resultados de investigaciones empíricas seleccionando finalmente 50 artículos. A continuación se hace un recorrido por las variables objeto de estudio, con las cuales se aborda el análisis.

\section{MÉTODO}

Para la selección de las investigaciones publicadas acerca del tema problema, se realizó un rastreo inicial con las palabras clave "estilos docentes," "estilos de enseñanza" "procesos atencionales, permitiendo indagar sobre relaciones existentes, contradicciones y diferencias encontradas con respecto a la literatura que ha sido publicada.

En cuanto a las base de datos seleccionadas, inicialmente se consultaron cuatro bases de datos, sin embargo, a medida que se fueron leyendo y analizando los artículos se incorporaron otras bases de datos, teniendo siempre en cuenta que dicho rastreo, como dice Morán (2008), se hizo sin la intención de ser un trabajo conclusivo, puesto que el educativo es un campo en constante cambio y actualización, este escrito pretende aportar elementos nuevos para la elaboración de las experiencias formativas.

Para demostrar el proceso elaborado en el rastreo de la información, se presenta el gráfico 1 , que de manera resumida da cuenta del proceso seguido en las bases de datos seleccionadas. 


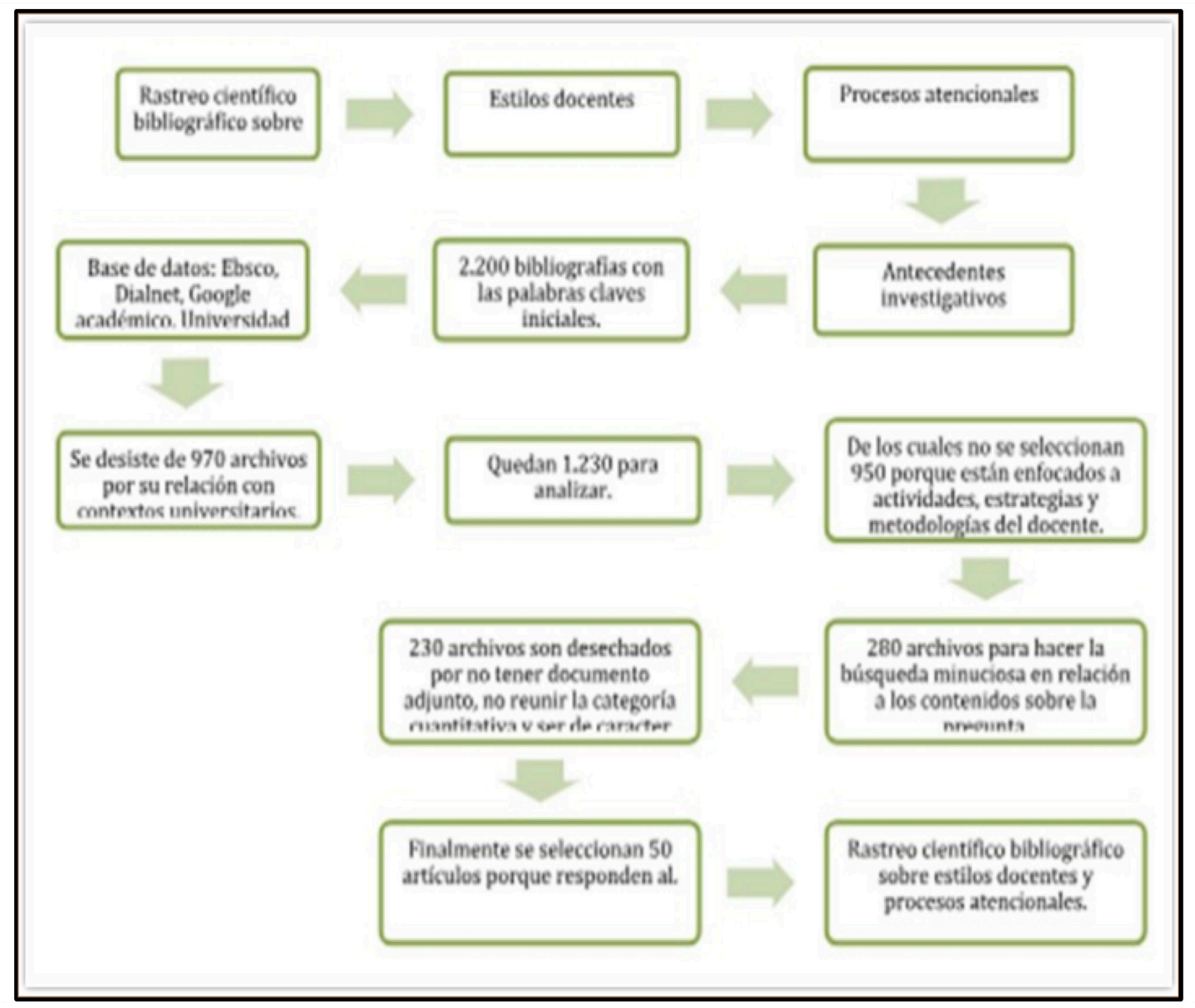

\section{Figura 1. Procesos de rastreo en bases de datos (elaboración propia).}

Tal como se evidencia en la figura 1, La búsqueda general sobre las bases de datos se constituyó en 2,200 referentes rastreados, de los cuáles se descartan 970 archivos porque su foco de investigación se centra en el campo de la salud o médico. Luego, los 1,230 textos restantes se revisan nuevamente, descartando aquellos donde la población se encontraba fuera de la etapa escolar. Se excluyen 950 textos, porque no se encontró coherencia entre el título de los artículos y el contenido, teniendo en cuenta las variables de nuestro estudio. Finalmente se descartan 280 artículos por no presentar el documento completo, seleccionando así, 50 archivos que tienen estrecha relación con el tema estilos docentes y procesos atencionales.

\section{RESULTADOS Y DISCUSIÓN}

Luego de la revisión de los artículos seleccionados, se analizó y clasificó dicha información en tres grandes categorías: estilos de enseñanza, estilos de aprendizaje y procesos atencionales sobre las cuales se sustenta el presente artículo. 


\section{Estilos de enseñanza}

En el rastreo de las teorías sobre estilos de enseñanza se encuentran diferentes propuestas que los clasifican de acuerdo a los múltiples criterios y objetivos que persigue cada autor a la hora de abordar el tema. A continuación, se presentan algunos de los autores que han hecho referencia al concepto.

Se puede considerar que los estilos de enseñanza están relacionados con la actividad docente lo cual exige estar dotado de una serie de habilidades, características personales y conocimientos que se hacen evidentes en la cotidianidad mediante las estrategias utilizadas, la calidad de la información socializada, su método de enseñanza incidiendo significativamente en la motivación y el rendimiento académico.

Como afirma Gómez (2017), el estilo de enseñanza depende tanto de la experiencia como de la formación previa y este favorece el desarrollo del trabajo docente, sus relaciones interpersonales, y mayor aprendizaje por parte de los estudiantes. A su vez, se afirma que los estilos de enseñanza son fundamentales para identificar las competencias y habilidades necesarias para proporcionar una educación completa y equilibrada a los estudiantes, además, estos estilos son influenciados por los valores personales (Di Loreto, 2014).

Sin embargo, el estilo de aprendizaje no actúa como mediador determinista/directo de un estilo de enseñanza concreto, debido a que puede recursivamente ser planificado, conducido y revisado por el estilo de enseñanza actual (Ventura, 2016). Por otro lado, al analizar el rendimiento académico como el sistema que mide logros y construcción del conocimiento en los estudiantes, se reconoce que estos logros o desempeños son mediados por intervenciones didácticas, educativas y evaluadas a través de métodos cualitativos y cuantitativos (Erazo, 2012).

En cuanto a la incidencia de la relación entre estilo de enseñanza y rendimiento académico en el contexto educativo, Pérez, Guerra, y Martínez, (2016), afirma que los estilos con mayor preferencia son el funcional, abierto y formal, debido a que generan resultados semejantes en el desempeño de los estudiantes. Respecto a los estilos de enseñanza de mando directo y los estilos de enseñanza de resolución de problemas, se evidencian diferencias significativas, al considerar al docente como aquel profesional reflexivo que realiza una labor de mediación entre el conocimiento y el aprendizaje de sus estudiantes (Duarte, 2017).

Así mismo, el rendimiento académico tiene una estrecha relación con las estrategias de aprendizaje y con el liderazgo docente, por lo que los docentes tienen un papel fundamental a la hora de enseñar a sus estudiantes a desarrollar sus propias estrategias de aprendizaje (Barragán, Pérez, Martos et al., 2016).

Consecuente con lo anterior, los estilos del docente se relacionan con la forma en que el docente presenta ante sus estudiantes una realidad o conocimiento, impregnado de intención, con fines y estrategias claras, y con un componente subjetivo como lo son sus expectativas en el incremento de la motivación y rendimiento académico de sus estudiantes, lo que implica un componente emocional importante a la hora de la situación de aprendizaje. Por lo tanto, para impactar positivamente en las emociones y mejorar el compromiso de los estudiantes, es relevante modificar el comportamiento y la motivación docente (Chiang, Díaz y Rivas, 2013; Renés y Martínez 2016; Sanzana, 2014; Valdivieso et al., 2014; Sánchez, Takaya y Molinari, 2016).

Se sustenta entonces, la importancia de la utilización de diferentes y variados estilos de enseñanza para responder a la pluralidad de los estudiantes, incluyendo en las programaciones docentes algunas variables relacionadas con los estilos de aprendizaje (Beltrán et al., 1987; Bennett, 1979; Flanders, 1985; Martínez-Geijo, 2007; Gregorc, 1985, citado en Carbonero, 2010). Todo ello, tomando en cuenta que el 
papel esencial del profesorado en la enseñanza es proveer al estudiante de múltiples bases de conocimiento, oportunidades de aprendizaje, recursos humanos, materiales y perspectivas diferentes de un mismo tema (Beltrán y Genovard, 1996, citado en Carbonero, 2010).

Así pues, los estilos de enseñanza se pueden clasificar de acuerdo a las características del docente desde un paradigma constructivista, en: funcional, donde se otorga mayor valor a los contenidos procedimentales y prácticos favoreciendo en el estudiante un estilo de aprendizaje relacionado con lo tangible. Estructurado, cuando el docente imparte los contenidos integrados a un marco teórico, amplio, articulado y sistemático, convirtiendo el proceso de enseñanza en un proceso con un orden secuencial y coherente. Formal, característico de los docentes que llevan una planeación detallada y se interesan por favorecer en el estudiante el aprendizaje reflexivo; y el estilo abierto el cual propone experiencias pedagógicas con actividades novedosas promoviendo en el estudiante el aprendizaje activo (Renés y Martínez, 2016; Chiang, 2013). Cada uno de los estilos de enseñanza planteados previamente presenta características que le son propias y se relacionan de forma directa con los estilos de aprendizaje de los estudiantes.

Es necesario destacar que existe otro estilo de enseñanza: el innovador, el cual hace presencia significativa en docentes de manera conjunta, ya que sus elementos adicionales se complementan, afianzando la interacción y permitiendo la realización de las tareas de enseñanza en varios niveles, en los que se evidencia la capacidad de permitir que el estudiante defiende la opción de elegir entre diversas actividades (Mosston y Ashworth, 1993; Piéron, 1988, citado en Isaza, 2012).

Finalmente, existe evidencia sobre la existencia de un estilo mixto de enseñanza, el cual resulta ser el más conveniente para atender las necesidades educativas de los estudiantes y los distintos ritmos y estilos de aprendizaje, puesto que la práctica docente no debe presentarse ignorando las características individuales y de contexto de los estudiantes (González y Pino, 2014).

\section{Estilos de aprendizaje}

El estudio de los estilos de aprendizaje sigue despertando el interés desde los ámbitos educativos, psicológicos y en la administración de recursos humanos de diversas empresas públicas o privadas. En los contextos educativos, el estilo de aprendizaje involucra habilidades como el pensar, aprender, analizar, procesar información, recordar y memorizar, las cuales permiten estructurar y mejorar el proceso de enseñanza y aprendizaje (Silva, 2018).

Con respecto a la relación entre los estilos de aprendizaje y estilos de aprendizaje, la misma se convierte en oportunidad para desarrollar la práctica pedagógica, y por ende las capacidades cognitivas del estudiante (Zambrano, Arango y Lezcano, 2018). Es aceptado que estos estilos muchas veces son utilizados de forma inconsciente tanto por docentes como por estudiantes, haciéndose necesario entonces crear los mecanismos que propicien tal conciencia, con el fin de lograr una mayor asociación entre ellos y hacer más eficientes los procesos de enseñanza y aprendizaje (Oviedo, 2010).

Un punto importante al hablar de eficiencia en los procesos de enseñanza y aprendizaje lo constituyen las prácticas docentes y la enseñanza de estrategias de aprendizaje, donde el aprendizaje colaborativo y la enseñanza de acuerdo a las necesidades individuales o grupales del estudiante es prioridad (Sanzana, 2014), por lo tanto el método del docente se convierte en una secuencia de acciones, actividades $u$ operaciones, las cuales expresan la naturaleza académica para el logro de los objetivos de enseñanza (Navarro y Samón, 2017).

De acuerdo al planteamiento anterior, es muy importante para el profesor utilizar 
metodologías didácticas variadas en el aula para que todos los estudiantes tengan la oportunidad de aprender, pues el docente se establece como facilitador del aprendizaje (Chiang, Díaz y Rivas, 2013; Renés y Martínez, 2016). En esta misma línea, Isaza y Henao (2012), afirman que los docentes constituyen un componente esencial y determinante en los procesos de enseñanza y aprendizaje, y que sus acciones y verbalizaciones atraviesan todas las situaciones educativas encaminadas a generar cambios y aprendizajes en los estudiantes. Enseñar, supone entonces, una reflexión integral, y se espera que en cada momento se evalúe la forma de hacer, lo cual requiere del docente una aptitud y una actitud artística, ya que en cada experiencia de socialización educativa se relacionan entre sí los espacios, el contexto, el tiempo, demanda una capacidad intuitiva y creadora para que el hecho de enseñar no les sea ajeno (Bruce, Weil y Calhoun, 2002, citado en Chiang, Díaz y Rivas, 2013).

\section{Procesos atencionales}

La atención se define como el mecanismo implicado directamente en la recepción activa de la información, donde la activación exitosa de los mecanismos atencionales entra a depender de las estrategias que docentes y estudiantes utilicen para fortalecer y potenciar los procesos atencionales en la vida escolar. Por tal razón, es conveniente concebir la atención como la capacidad de seleccionar la información sensorial en todos los momentos de la vida diaria y de dirigir los procesos mentales, lo que implica un proceso de control y ejecución de la acción (Llorente, Oca y Solana, 2012).

La atención y el actuar como un mecanismo activo y constructivo se modifica con la práctica, generando en cada sujeto un potencial atencional propio, que no solamente va a estar determinado por elementos cognitivos, sino también por elementos conativos y afectivos, a través de la práctica continuada, donde se involucran diferentes mecanismos que trabajan de forma conjunta y coordinada (Holgado y
Alonso, 2015; Cristóforo, Delgado, Valazza y Pou, 2013).

Lo anterior, implica un compromiso comportamental del estudiante, con capacidad de influir en los resultados educativos; comportamiento que es mediado por las emociones y por sus antecedentes contextuales e individuales, lo cual influye significativamente en el proceso de enseñanza y aprendizaje. $Y$ es precisamente en términos de enseñanza, donde la cognición tiene un efecto singular a partir de las tecnologías, derivado de las TICs a través del diseño, la actividad y el entorno, en función de fomentar la abstracción consciente de habilidades y estrategias, en función de la atención (Sánchez, Maquilón y Cuesta 2016; Garay y Keegan, 2015; Etcheverry, Calixto Maldonado (2014).

Por otro lado, los desórdenes atencionales son factores de riesgo que inciden en el rendimiento académico y social de los estudiantes, adquiriendo importancia evaluar las habilidades atencionales con instrumentos válidos, confiables y adaptados a la población escolar, reconociendo la importancia de las evaluaciones complementarias como soporte al diagnóstico para proponer planes de intervención que permitan afianzar las habilidades de los estudiantes de acuerdo a sus posibilidades (Monteoliva, 2014; Holgado y Alonso, 2015).

Específicamente, uno de los instrumentos válidos en poblaciones de niños entre 4 y 6 años, es la Bateria Neuro Psicológica Luria Inicial (BLI), cuyos resultados en diversas investigaciones han demostrado su pertinencia al evaluar las condiciones preacadémicas del niño con y sin alteraciones del neurodesarrollo antes de comenzar la escolaridad (Ramírez, Díaz, Somoano y Hernando, 2011).

En cuanto a la relación entre los procesos atencionales y el rendimiento académico, variables como la exploración, subrayado lineal, fragmentación y atención, pareciesen influir más en el rendimiento académico, sin embargo, se evidencia un ligero desuso 
de estrategias atencionales a lo largo de la educación secundaria sobre todo en los estudiantes masculinos (Tejedor, González y García, 2008). A sí mismo, se evidencia que la dificultad en la ejecución de una tarea cognitiva que involucra operaciones atencionales no es necesariamente un índice de existencia de un problema atencional, planteamiento que cobra relevancia en los contextos educativos donde muchas veces se diagnóstica o etiqueta de manera apresurada y errónea ciertas dificultades en el desarrollo cognitivo (Carrada e Ison 2013).

En este aspecto Ruggieri (2006), se refiere a la atención como un proceso integral y relacionado con procesos de selección, los cuales permiten identificar y separar la información y el análisis de los diversos componentes atencionales como alerta, orientación, mirada, filtrado, atención sostenida y cambios de atención, y solo así se podrá relacionar estos con trastornos por déficit de atención. A su vez, resulta evidente el valor de la atención en la creatividad, dando relevancia a programas basados en la adquisición de la información, los cuales puede tener efectos positivos en el pensamiento creativo (Cárdenas, López y Arias, 2018).

Una de las condiciones que afecta la atención e interfiere en el proceso del aprendizaje del lenguaje, es el desempeño en la comprensión lectora y producción de textos en los estudiantes con déficit atencional (Inostroza, 2017), convirtiéndose entonces, en un gran reto para el docente a la hora de planificar y adaptar la enseñanza en el área del lenguaje y la lectoescritura en estudiantes con y sin dicha condición.

La capacidad de atención y el rendimiento académico tienen un incremento significativo al entrenar las habilidades cognitivas como la memoria de trabajo, lo que evidencia que el rendimiento académico está ligado a factores como la inteligencia y funciones ejecutivas, las cuales puede mejorar si se consigue incrementar la capacidad de memoria de trabajo (Sivó, 2016; Monteoliva, Carrada e Ison,
2017). La capacidad atencional es clave para un adecuado funcionamiento cognitivo y tiene una correlación positiva con competencias como la lectura, escritura y habilidades matemáticas en el ámbito escolar.

Al analizar la relación entre el fracaso escolar, las estrategias de aprendizaje y el liderazgo docente en relación con el rendimiento académico, se encuentra que existe relación con variables predictoras como la violencia entre iguales y los estilos parentales (Barragán, et al. 2016), haciendo necesario la adquisición de la capacidad de liderazgo por parte de los docentes para desarrollar en los estudiantes estrategias de aprendizaje, sin perder de vista que dentro de los procesos atencionales, la atención selectiva es la que permitirá el procesamiento de la información relevante mientras (Ballesteros, 2014).

\section{Relación estilos de enseñanza, estilos de aprendizaje y procesos atencionales}

El estilo de enseñanza se origina a partir de la interacción entre docente y estudiante, y en ella, ambos se manifiestan como una unidad, es decir, el estilo de enseñanza influye en el estilo de aprendizaje y viceversa, evolucionan y se dinamizan en la medida que existan estilos de enseñanza que efectivamente promuevan dicha evolución (Rendón, 2013; Navarro y Samón, 2017; Chiang, Díaz y Rivas, 2013; Soler, Cárdenas, Hernández y Monroy, 2017).

Partiendo de la aceptación a la diversidad, se comprende que el estilo de enseñanza y el estilo de aprendizaje, se constituyen en la habilidad que posee cada persona frente a una forma de enseñar y de aprender, que lo caracteriza de forma individual, y que se manifiesta en una variedad de conductas y roles a lo largo del proceso educativo, logrando con esto consolidarse en un estilo determinado (González, y Pino, 2014).

Estilo, que se resumen en los métodos de enseñanza y los métodos de aprendizaje, con diferentes intenciones, diferentes perspectivas 
y que se proyectan hacia resultados diferentes. Es, entonces, el método de enseñanza la secuencia de acciones, actividades u operaciones del que enseña, que coloca en evidencia las formas académicas en la organización del proceso de enseñanza, mientras que el método de aprendizaje se constituye también en una secuencia de acciones, actividades u operaciones del que aprende, pero quien aprende atiende a la estructura interna de la forma académica, pero se expresa dentro y fuera de esta, procesando, integrando, adquiriendo y asimilando el contenido de enseñanza que provoca cambios en su sistema de conocimientos y su conducta (Navarro y Samón, 2017).

Por lo tanto, el aprendizaje está directamente relacionado con las prácticas docentes y las estrategias de aprendizaje por parte de los estudiantes, reconocido como ejercicio de corresponsabilidad, entre docente y estudiante con claras estrategias de elaboración y organización (López-Iñiguez y Pozo, 2014, citado en Ventura, 2016). Sin embargo, la incidencia de la formación continuada en las prácticas docentes y el efecto que esta tiene en la mejora de los aprendizajes de los estudiantes no siempre es lineal, ni evidente, una muestra de ello es que los docentes suelen valorar más positivamente la incidencia en sus aprendizajes que en el de los mismos estudiantes (González y Cutanda, 2017).

De acuerdo a lo anterior, el enfoque de enseñanza involucra diversas maneras de actuar en el aula, fundamentadas en principios pedagógicos e ideológicos que reconocen determinadas concepciones sobre la enseñanza y la educación (Carr, 1986). Es aquí donde la figura docente es clave para los procesos cognitivos en la educación inicial, debido a que en estas edades se observa una capacidad planificadora y atencional por una mayor competencia cognitiva a la vez que se manifiesta una relación entre ambas (Martínez y Páramo, 2015).

La determinación de los estilos cognitivos, relacionados con los procesos de aprendizaje contribuyen a orientar en los estudiantes los patrones de conceptualización y de pensamiento, más aún cuando se sabe que las dificultades en los logros escolares se deben también a los desórdenes de tipo sensorial y perceptual que impiden el almacenamiento de la información que se debe aprender (Ovalle y Tobón, 2017).

Por otro lado, lo expuesto anteriormente invita a pensar sobre la estrecha relación que puede llegar a existir entre el estilo docente y los procesos atencionales considerándose que el estilo docente favorece la creatividad, y se convierte en componente esencial y determinante en los procesos de enseñanza y aprendizaje. El docente con un elevado sentimiento de autoeficacia muestra mayor apertura a nuevas ideas, está más dispuesto a probar métodos nuevos de enseñanza, sus acciones y verbalizaciones intervienen en todas las situaciones educativas encaminadas a generar cambios y aprendizajes en los estudiantes (Valdivieso et al., 2014).

La Madriz (2013), afirma que en el ámbito académico, la comunicación efectiva se perfila como una herramienta didáctica que empleada adecuadamente por docentes y estudiantes, potencia el proceso de enseñanza y aprendizaje, en la medida que eleva el nivel de comprensión entre estos, logra aumentar la participación del estudiante y da un nuevo protagonismo al docente al no ser ya el orador retórico, sino el conductor de las disertaciones que se manifiesten entre los educandos y entre estos y él. Para Montes, Caballero y Miranda (2017), las prácticas docentes, se pone en el centro a las acciones del profesor, con prevalencia.

\section{DISCUSIÓN}

La relación existente entre los estilos de enseñanza, estilos de aprendizaje y procesos atencionales, considerándolos componente esencial y determinante en los procesos de enseñanza y aprendizaje en el ámbito escolar ha sido poco explorada. Al igualar el término estilo con método, forma, enfoque y proceso de 
enseñanza y aprendizaje, evidencia que dicha relación está atravesada por la personalidad, el comportamiento y el conocimiento sobre la enseñanza del docente, el aprendizaje y los procesos atencionales del estudiante, donde el docente actúa como mediador entre el conocimiento y el aprendizaje.

Tal como se observó en el análisis realizado, existe relación entre estilo de enseñanza y rendimiento académico en el contexto educativo, se muestra cómo algunos estilos de enseñanza tienen mayor preferencia y un mayor impacto en la generación de resultados en el desempeño de los estudiantes. Se sostiene la importancia de los estilos de enseñanza para el aprendizaje, pues estas responden a la pluralidad de los estilos de aprendizaje de los estudiantes, suministrando variadas bases de conocimiento y perspectivas diferentes de un mismo tema.

A su vez, se encuentra coincidencia entre los estilos de enseñanza relacionados con la actividad docente dotada de habilidades y las características personales que incide en la motivación y el rendimiento académico, considerándose un componente esencial y determinante en los procesos de enseñanza y aprendizaje, y por lo tanto, generador de cambios.

Por otro lado, el estilo de aprendizaje se asocia a aspectos educativos, donde se involucran habilidades cognoscitivas fundamentales para el aprendizaje, y son vistos como una oportunidad para potenciar la práctica pedagógica desde su reconocimiento. El conocer y determinar los estilos de aprendizaje existentes, permite estructurar y mejorar el proceso de enseñanza y aprendizaje, y pudiendo ser más efectivo.

Se considera que la atención y el actuar, se modifica a través de la práctica continuada, determinada por elementos cognitivos, conativos y afectivos, siendo la atención un compromiso comportamental del estudiante. Algunos autores reconocen que la evolución y dinámica de los estilos de enseñanza y aprendizaje dependen de la interacción entre docente/estudiante.

\section{CONCLUSIONES}

Después de realizar el ejercicio de revisión y análisis, se encuentra en un primer momento que las variables seleccionadas en el presente artículo han sido objeto de recientes investigaciones en lengua española a nivel internacional y nacional, sin embargo no se evidencia consenso unificado en la denominación de las mismas, por ejemplo para el caso de estilos de enseñanza se encuentra como forma de enseñanza, métodos de enseñanza y procesos de enseñanza; en cuanto a estilos de aprendizaje otros autores la nombran como métodos de aprendizaje, formas de aprendizaje, procesos de aprendizaje y estilos cognitivo.

En cuanto a los procesos atencionales, estos han sido nombrados como niveles atencionales, trayectorias atencionales y procesos cognitivos, esta forma de nombrarlas sesgó y limitó inicialmente el rastreo que se llevaba a cabo.

\section{REFERENCIAS}

Álvarez, L., González, P., Núñez, J., González, J., Álvarez, D., y Bernardo, A. (2007). Desarrollo de los procesos atencionales mediante «actividades adaptadas. Revista Papeles del Psicólogo, 28 (3), 211-217.

Ballesteros, S. (2014). La atención selectiva modula el procesamiento de la información y la memoria implícita. Revista Acción Psicológica, 11(1), 7-20.

Barragán, A., Pérez, M., Martos, Á., Simón, M., Molero, M., y Gázquez, J (2016). Intervención y variables del personal docente y el centro escolar que modulan el rendimiento académico del alumno. Revista de Psicología INFAD, 5 (1), 387-396.

Carbonero, M., Martín, L., Román, J., y Reoyo, N. (2010). Efecto de un programa de 
entrenamiento al profesorado en la motivación, clima de aula y estrategias de aprendizaje de su alumnado. Revista Iberoamericana de Psicología y Salud, 1(2), 117-138.

Carbonero, M., Román, J., Martín, L., Valdivieso J., Reoyo, N. y Freitas, A. (2014). Habilidades docentes y estilos de afrontamiento en profesores de educación primaria. Revista INFAD Revista de Psicología, 5 (1), 361-370.

Cárdenas, N., López, V., y Arias, C. (2018). Análisis de la relación entre creatividad, atención y rendimiento escolar en niños y niñas de más de 9 años en Colombia. Revista Psicogente, 21 (39), 35-49.

Carrada, M., Ison, M. (2013). La eficacia atencional. Un estudio normativo en niños escolarizados de Mendoza. Revista Latinoamericana de Ciencia Psicológica Psiencia, 5 (2), 63- 73.

Castro, M., Paternina M., Argelis B., Gutiérrez B., y Mailen R. (2014). Factores pedagógicos relacionados con el rendimiento académico en estudiantes de cinco instituciones educativas del distrito de Santa Marta, Colombia. Revista Intercontinental de Psicología y Educación, 16 (2), 151-169.

Cristóforo, A., Delgado, R., Valazza, V., y Pou, V. (2013). La Función Atencional en niños que concurren a una escuela de contexto socio económico muy desfavorable. Revista Psicología, Conocimiento y Sociedad 2 (3), 5 -30 .

Chiang, M., Díaz, C., y Rivas A. (2013). Un cuestionario de estilos de enseñanza para el docente de Educación Superior. Revista Lasallista de Investigación, 10 (2), 62-68. Colombia.

Di Loreto, M. (2014). Enseñanza en la educación primaria y valores: diferencias entre España e Italia. Revista de Psicología INFAD, 2(1), 51-61.

Duarte, J. (2017). Diferencias de los estilos de enseñanza: Mando directo y Resolución de problemas en el resultado para la enseñanza de la cualidad física de fuerza de tren inferior. Revista Ciencias de la Actividad Física, 18 (2), 49-58.

Etcheverry, P.; Maldonado, C. (2014). Investigación Mixta del Proceso Cognitivo Atención en la Interfaz de aprendizaje. Workshop de Investigadores en Ciencias de la Computación, 1123 - 1158.

Erazo, O. (2012). El rendimiento académico, un fenómeno de múltiples relaciones y complejidades. Revista Vanguardia Psicológica Clínica Teórica y Práctica, 2 (2), 144-173.

Garay, C., y Keegan E. (2016). Terapia metacognitiva. El síndrome cognitivo atencional y los procesos cognitivos. Revista Argentina de Clínica Psicológica, 2,125-134.

Gómez, J. (2017). Nuevos estilos de enseñanza en la era de la convergencia tecnomediática: hacia una educación holística e integral. International Journal of Educational Research and Innovation (IJERI), 8, 60-78.

González, M. (2013). Los estilos de enseñanza y aprendizaje como soporte de la actividad docente. Revista Estilos de Aprendizaje, 11 (11), 1-23.

González, M., y Cutanda, M. (2017). Formación continuada del profesorado, mejora de la enseñanza y de los aprendizajes del alumnado. Revista mexicana de investigación educativa, 22 (75), 1095-1116.

González, M., Pino, M. (2014). Aproximación a las Representaciones y Creencias del Alumnado de Magisterio Sobre los Estilos de Enseñanza. Educación XXI, 17 (1), 83-110.

Holgado, B., y Alonso, L. (2015). Evaluación de un programa psicopedagógico para la mejora de la atención en estudiantes de educación primaria. Revista Española de Orientación y Psicopedagogía, 26 (3), 26-44.

Inostroza, F. (2017). Estudiantes con déficit atencional enfrentando tareas de comprensión 
lectora y producción de textos: Un estudio comparativo en alumnado de $4^{\circ}$ año de educación primaria en Chile. Revista Electrónica Educare, 21(3), 1 - 20.

Isaza, L., y Henao G. (2012). Actitudes/ estilos de enseñanza: su relación con el rendimiento académico. International Journal of Psychological Research, 5 (1), 133-141.

Krüger, K. (2006). El concepto de sociedad del conocimiento. Revista Bibliográfica de Geografía y Ciencias Sociales, XI (683)

La Madriz, L., y La Madriz, J. (2013). Comunicación efectiva como estrategia didáctica para potenciar el proceso de enseñanza y aprendizaje. Revista Educare, 17(1), 74-92.

Llorente, C, Oca, J., y Solana, A. (2012). Mejora de la atención y de áreas cerebrales asociadas en niños de edad escolar a través de un programa neurocognitivo. Revista del consejo escolar del estado, 47 (56) 12-30

Martínez, M., y Páramo, M. (2015). Una Mirada a los Procesos Cognitivos de Atención y Planificación en el Alumnado en Educación Infantil. Revista Iberoamericana de Evaluación Educativa, 8(1), 26-40.

Monteoliva, J., Carrada, M., e Ison, M. (2017). Test de percepción de diferencias: Estudio normativo del desempeño atencional en escolares argentinos. Revista Interdisciplinaria, 34(1), 39-56.

Monteoliva, J., Valdivieso, J., Reoyo, N. (2014). Evaluación del desempeño atencional en niños: Eficacia, eficiencia y rendimiento. Revista Interdisciplinaria, 31(2), 213- 226.

Montes, L., Caballero, T., y Miranda, M. (2017). Análisis de las prácticas docentes: estado del conocimiento en DOAJ y EBSCO (2006-2016). Revista de Investigación Educativa, (25) 197-229.

Montes, A., Gamboa, A., y Suárez, C. (2013). La Educación Básica en Colombia: Una mirada a las Políticas Educativas. Revista Saber, Ciencia y Libertad, 8 (2), 143-157.

Morán, L. (2008). Criterios para análisis comparativo de modelos y diseños educativos. Revista Educación y Educadores, 11(2),139158.

Navarro, D., y Samón, M. (2017). Redefinición de los conceptos método de enseñanza y método de aprendizaje. Revista EduSol, 17 (60).

Ovalle, A, A. y Tobón, G. (2017). Calidad de la interacción docente-niño en el aula y los estilos cognitivos en la dimensión reflexividadimpulsividad. Revista Latinoamericana de Estudios Educativos, 13 (1), 201-222.

Oviedo, P., Cárdenas, F., Zapata, P., Rendón, M., Rojas, Y., y Figueroa, L. (2017). Estilos de enseñanza y estilos de aprendizaje: implicaciones para la educación por ciclos. Revista Actualidades Pedagógicas, (55), 31-43.

Pérez, O., Guerra, E., y Martínez, P. (2016). Estilos de enseñanza y rendimiento académico. Revista de Estilos de Aprendizaje, 9 (18).

Ramírez, Y., Díaz, M., Somoano, R., y Hernando, D. (2011). Batería Neuropsicológica Luria Inicial y procesos atencionales. Revista chilena de neuropsicología, 6 (9) 1-6.

Rendón, M. (2013). Hacia una conceptualización de los estilos de enseñanza. Revista Colombiana de Educación, (64), 175-195.

Renés, P., y Martínez, P. (2016). Una mirada a los estilos de enseñanza en función de los estilos de aprendizaje. Revista de Estilos de Aprendizaje, 9 (18), 224-243.

Ruggieri, V. (2006). Procesos atencionales y trastornos por déficit de atención en el autismo. Revista de Neurología, 42 (3), S1-56.

Sánchez, J., Takaya, P., y Molinari, A., (2016). Atención en clase: rol predictivo del comportamiento docente, valor de la tarea, 
autoeficacia, disfrute y vergüenza. Revista PSIENCIA. Revista Latinoamericana de Ciencia Psicológica, 8(3) doi: 10.5872/psiencia/8.3.22

Silva, A. (2018). Conceptualización de los Modelos de Estilos de Aprendizaje. Revista de Estilos de Aprendizaje, 11(21), 1-33.

Sivó, P. (2016). Efecto del entrenamiento de la memoria de trabajo en los procesos atencionales, en el rendimiento académico y las funciones ejecutivas y memoria de trabajo en niños/as de entre 4/6 años (tesis doctoral). Universidad Castilla La Mancha. Departamento de Psicología. España.

Sánchez, M., Maquilón, J., y Cuesta, S. (2016). Enseñar y aprender en las aulas de Educación Primaria. REDIE. Revista Electrónica de Investigación Educativa, 18 (2), 144-155.

Soler, M., Cárdenas, F., Hernández, F., y Monroy, F. (2017). Enfoques de aprendizaje y enfoques de enseñanza: origen y evolución. Revista Educación y Educadores, 20 (1), 65-88.

Tejedor, F., González, S., y García, M. (2008). Estrategias atencionales y rendimiento académico en estudiantes de secundaria. Revista Latinoamericana de Psicología, 40(1),
123-132.

Valdivieso, J., Reoyo, N., Martín, L., Carbonero, M., y Freitas, A. (2014). Estilos de enseñanza y autoeficacia docente en educación primaria. Revista Infad de Psicología. 5(1), 387395

Ventura, A. (2016). ¿Enseño cómo aprendí?: el rol del estilo de aprendizaje en la enseñanza del profesorado universitario. Revista Aula Abierta, 44 (2), 91-98

Zambrano, J., Arango, L., y Lezcano, M. (2018). Estilos de aprendizaje, estrategias de aprendizaje y su relación con el uso de las tic en estudiantes de educación secundaria. Revista de Estilos de Aprendizaje, 11(21), 130-159.

Carr, K (1986). Teoría crítica de la enseñanza. La investigación/acción en la formación del profesorado. Barcelona, España: Ediciones Martínez Roca.

Tesis Doctoral:

Sanzana, G. (2014). La práctica de aula: percepción de efectividad y autoeficacia. Universidad de Córdoba. España. 